\title{
O Não-dito da AIDS
}

\section{The Hidden Face of AIDS in Brazil}

\section{Francisco Inácio Bastos ${ }^{1}$; Cynthia Boschi-Pinto ${ }^{2}$; Paulo Roberto Telles ${ }^{1} \&$ Elson Lima ${ }^{1}$}

\begin{abstract}
BASTOS, F. I.; B OSCHI-PINTO, C.; TELLES, P. R. \& LIMA, E. The Hidden Face of AIDS in B razil. Cad. Saúde Públ., Rio de J aneiro, 9 (1): 90-96, jan/mar, 1993.

The authors report on the evolution of the AIDS epidemic in B razil from the point of view of the construction of social representations about "risk groups" involved in the spread of the disease. They emphasize the need to demystify the immediate correlation between AIDS and homosexual transmission. They highlight the role of intravenous drug users and of heterosexual transmission in new AIDS cases in Brazil - groups and behaviours that are not included in the priorities of local health authorities.
\end{abstract}

Keywords: AIDS; Heterosexual Transmission of HIV; HIV Transmission among Injecting Drug U sers (IDUs)

\section{INTRODUÇÃO}

Como todo fenômeno novo, complexo e ameaçador, a AIDS vem sendo objeto de uma série de visões reducionistas que, ao mencionarem aspectos parciais da questão, estigmatizam segmentos da população, impedem uma visão realmente abrangente e integrada do problema e restringem os esforços de prevenção aos grupos previamente apontados como em risco de contrair a doença.

Historiamos, a seguir, brevemente, algumas dessas visões relativas a grupos sucessiva ou concomitantemente chamados ao primeiro plano das preocupações, de modo geral, infelizmente, em função do preconceito.

Ao longo do tempo, estes grupos vêm explicitando suas posições a partir das reações ao estigma e da sua auto-organização grupal.

Além disso, estudos acadêmicos, ao desconstruirem o imediatismo e a circularidade das visões preconcebidas, têm aberto caminho para

\footnotetext{
${ }^{1}$ Núcleo de Estudos e Pesquisas em A tencão ao U so de Drogas da Universidade Estadual do Rio de Janeiro. Rua Fonseca Teles, 121, 40 andar, 20940-200, Rio de Janeiro, RJ, Brasil.

${ }_{2}$ Centro de Informações para a Saúde da Fundação Oswaldo Cruz. Av. Brasil, 4365, 21045-900, Rio de Janeiro, RJ, Brasil.
}

o enfrentamento efetivo das questões.

É inequívoco que a epidemia, entre nós, vem mudando de perfil. No entanto, estas mudanças, ao nosso ver, não vêm sendo objeto da necessária atenção e tampouco vêm sendo enfrentadas adequadamente.

Nosso objetivo neste trabalho é colocar em foco dois segmentos que vêm tendo participação crescente tanto na transmissão do HIV quanto nos novos casos da doença.

O primeiro segmento abordado é o dos usuários de drogas pela via injetável (UDI), segmento que, em função do caráter ilícito e da intensa estigmatização do seu comportamento, não tem, via de regra, sua problemática discutida de forma aberta e ampla.

O segundo, ao confundir-se com a própria população como um todo, provavelmente não pode nem mesmo ser considerado um segmento. Trata-se dos indivíduos contaminados pelo HIV através da transmissão heterossexual, a qual o senso comum tantas vezes julga rara ou inexistente e que surpreende os meios de comunicação, esporadicamente, como no caso do famoso jogador norte-americano de basquete.

\section{HISTÓRICO}

A AIDS começa a ser mencionada nos meios de comunicação brasileiros inicialmente como 
uma doença "vinda do exterior", basicamente dos Estados Unidos da América e, em especial, de Nova Iorque. A doença, de início, esteve sempre vinculada à homossexualidade, tendo sido chamada de "peste gay" ou "câncer gay" (Carrara 1987a, 1987b ). O primeiro caso fatal amplamente noticiado é o de um costureiro homossexual pertencente à classe alta, o que consolida a imagem da doença como proveniente, e exclusiva, de homossexuais de classe alta que freqüentemente viajavam para o exterior.

As autoridades sanitárias, à época (meados dos anos 80), declaravam que o problema não merecia uma atenção especial em virtude de, segundo a crença mais ou menos generalizada de então, restringir-se a grupos minoritários. Esta visão, comum a diversas autoridades sanitárias, se alimentava do contraponto constante entre a AIDS e as doenças ditas da pobre$\mathrm{za}$, como as grandes endemias.

Reagindo tanto ao risco efetivo como ao estigma, os diversos grupos de homossexuais começam a se mobilizar em torno da questão, iniciando a discussão sobre práticas de sexo seguro e combatendo o imediatismo da correlação AIDS-homossexualismo.

$\mathrm{Se}$, por um lado, isso fez com que uma parcela dentre os homossexuais - geralmente os pertencentes a uma fração com maior acesso às informações e/ou maior militância e participação comunitária - se prevenisse de forma mais eficaz ao tomar conhecimento de informações acerca das práticas do "sexo seguro", por outro, não atingiu a maioria da população em geral e mesmo a maioria dos próprios homossexuais, a princípio.

Apesar de uma intensa mobilização deste último grupo, ainda hoje persiste a idéia da relação unicausal AIDS-transmissão homossexual.

Ao longo dos anos 80 e início desta década, estruturaram-se diversas organizações nãogovernamentais voltadas para a difusão de informações sobre a prevenção, para a ajuda a pessoas portadoras do HIV, e para a fiscalização e crítica às alternativas governamentais, tanto no âmbito da assistência aos pacientes como na elaboração de estratégias de prevenção e ações no campo da educação para saúde.
A primeira organização a se estabelecer - o Grupo de Apoio e Prevenção à AIDS (Gapa) nasce em 1985, a partir do esforço conjunto de profissionais de saúde envolvidos mais diretamente na questão e militantes do movimento homossexual.

O significativo índice de contágio entre pacientes politransfundidos, em especial os hemofílicos, agudizou e explicitou de forma dramática o descaso em relação ao controle da qualidade do sangue e hemoderivados (Santos, 1991, 1992). As iniciativas vinculadas à questão do controle de sangue e derivados levaram à criação da Associação Brasileira Interdisciplinar de AIDS (Abia), em 1986, associação esta que, posteriormente, estendeu sua atuação para um amplo leque de ações preventivas, educativas e de fiscalização de políticas públicas nas áreas de prevenção e assistência (Vallinoto, 1991).

Ao lado da discussão sobre a transmissão da doença através do contato homossexual, houve crescente mobilização em torno da questão da qualidade do sangue e hemoderivados, com a apuração de diversas denúncias, fechamento de estabelecimentos e implementação de um melhor controle de qualidade (Santos, 1992).

O final dos anos 80 e início dos 90 testemunham uma série de estudos relativos à imagem e aos preconceitos vinculados à condição homossexual, que de forma mais ou menos mediata produzem, mesmo que num segmento restrito, com um nível cultural satisfatório e/ou maior engajamento, um remanejamento da correlação simplista AIDS-transmissão homossexual.

Estes trabalhos redefinem a condição homossexual face às especificidades sócio-culturais (Fry, 1982; Parker, 1991; Daniel, 1990), tematizam as próprias estratégias dos grupos homossexuais (McRae, 1990) e até mesmo buscam desconstruir o conceito de homossexualismo (Costa, 1991a, 1991b).

Estes anos assistem, igualmente, à criação de outras organizações não-governamentais de variados recortes sociais e propostas: Atobá, ARCA, Programa Prostituição e Direitos Civis, e o grupo Pela VIDDA. 


\section{DADOS SOBRE A EPIDEMIA NO BRASIL}

A epidemia de AIDS no Brasil teve início em 1982, com uma taxa de incidência acumulada de 0,05/100000 habitantes. Em outubro de 1992 esta taxa era igual a 23,7/100000 habitantes (MS, 1992), correspondendo à quarta posição em incidência acumulada na América do Sul (Bernard, 1992).

A epidemia vem mostrando tendência crescente a partir dos primeiros casos notificados (1980-1986) em todos os estados da Federação. Estes casos se concentram especialmente nos estados de São Paulo e Rio de Janeiro (Tabela 1), considerados portas de entrada da infecção no país, perfazendo $75,2 \%$ do total de notificações. Roraima, Distrito Federal e Rio Grande do Sul apresentam, respectivamente, a terceira, quarta e quinta maiores taxas de incidência em todo o Brasil (MS, 1992). Acredita-se que a elevada taxa de incidência encontrada em Roraima, cerca de sete vezes maior que a taxa média da Região Norte (3,8/100000 hab), devase à sua proximidade com as Guianas, que apresentam as mais elevadas taxas de incidência acumulada de toda a América do Sul (Bernard, 1992).

\section{O PAPEL DOS UDIS E DA TRANSMISSÃO HETEROSSEXUAL NA DISSEMINAÇÃO DO HIV}

Há um quadro de quase total silêncio acerca da relevância do grupo dos usuários de drogas injetáveis na transmissão do HIV, assim como do papel da transmissão do vírus através do contato heterossexual. Este silêncio traduz-se pela indefinição de uma política de prevenção especificamente dirigida aos UDIs e pela pouca ênfase dada ao papel da transmissão heterossexual.

Vejamos, primeiro, a questão do ponto de vista da transmissão por seringas e agulhas contaminadas, compartilhadas por usuários de drogas, embora não deva ser subestimado o papel da transmissão sexual entre os próprios UDIs e entre estes e seus parceiros/as.

Desde o início da epidemia de AIDS que o papel do uso injetável de drogas vem tendo crescente importância como um dos principais modos de transmissão do HIV.

Dentre os cinco países europeus com maior número de casos de AIDS (França, Alemanha, Itália, Espanha e Inglaterra), a proporção daqueles atribuíveis ao uso injetável de drogas varia

TABELA 1. Número de Casos de AIDS e Taxas de Incidência (100000 hab) Segundo Período de Diagnóstico e Local de Residência — Estados das Regiões Metropolitanas Brasileiras e Roraima $1980 / 1992$

\begin{tabular}{lcccccc}
\hline \hline \multirow{2}{*}{ Unidade Federada } & \multicolumn{2}{c}{$1980-1986$} & \multicolumn{2}{c}{1991} & \multicolumn{2}{c}{$1980-1992$} \\
& $\mathrm{~N}$ & Taxa & $\mathrm{N}$ & Taxa & $\mathrm{N}$ & Taxa \\
\hline Roraima & 0 & 0,0 & 10 & 8,2 & 27 & 27,0 \\
Pará & 3 & 0,1 & 44 & 0,9 & 156 & 3,8 \\
Ceará & 16 & 0,3 & 113 & 1,8 & 343 & 5,9 \\
Pernambuco & 27 & 0,4 & 147 & 2,0 & 617 & 9,2 \\
Bahia & 22 & 0,2 & 180 & 1,5 & 624 & 5,9 \\
Minas Gerais & 59 & 0,4 & 238 & 1,5 & 1137 & 7,9 \\
Rio de Janeiro & 388 & 3,3 & 908 & 6,6 & 4933 & 39,4 \\
São Paulo & 994 & 3,7 & 4997 & 15,1 & 18715 & 64,6 \\
Paraná & 20 & 0,3 & 237 & 2,8 & 737 & 9,2 \\
Rio Grande do Sul & 64 & 0,8 & 449 & 5,0 & 1468 & 17,5 \\
\hline \hline
\end{tabular}

Fonte: Ministério da Saúde. Aids. Boletim Epidemiológico, ano V, nº 7, 1992. 
de $4,4 \%$, na Inglaterra, a aproximadamente $65 \%$ dos casos, na Itália e Espanha (European Centre, 1991).

Nos EUA, até o momento, entre a população maior de 13 anos, 23\% de todos os casos notificados devem-se à contaminação em função do compartilhamento de seringas contaminadas (CDC, 1992).

Atualmente, no Brasil, a participação dos UDIs tem alterado de forma significativa o perfil da epidemia. Cabe notar que o grupo de risco UDIs reúne, na verdade, um conjunto de pessoas com hábitos, visões de mundo e atitudes perante os riscos de contaminação bastante heterogêneos (Schiller, 1992). A identificação deste conjunto de pessoas com a sua "extremidade" mais desinserida do ponto de vista social - os assim chamados usuários "junkiesados" (Baratta, 1992) - é antes fruto do preconceito e dos constrangimentos legais impostos sobre estas condutas. Esta simplificação obscurece a real dimensão do problema e torna urgentes estudos qualitativos que complementem os levantamentos epidemiológicos.

A participação percentual dos UDIs (enquanto fator simples) passou de apenas 3,0\% do total de casos notificados de AIDS, no período 1980-1986, para 24,9\%, em 1992 (MS, 1992).

Alguns estudos de soroprevalência realizados na cidade do Rio de Janeiro e no estado de São Paulo (Capital, Santos e Campinas) revelam prevalências significativas do HIV entre UDIs. Estas taxas de prevalência chegam a atingir $38 \%$ no Rio (Telles, 1992), 76\% em São Paulo/Capital (Gianna, 1989), 59\% em Santos (Mesquita, 1992) e cerca de 50\% em Campinas (Lima, 1989; Leite, 1989; Dias, 1989). Cabe notar que a maior parte dos estudos acima mencionados foi realizada em amostras de usuários atendidos em centros de tratamento e que, portanto, os resultados podem não ser aplicáveis ao restante da população de usuários de drogas não engajados em programas de tratamento.

Muito embora o número de indivíduos infectados pelo HIV, dentre os heterossexuais, tenha sido estimado entre 75000 e 125000 , apenas muito recentemente tem sido dada alguma atenção à transmissão heterossexual da AIDS por parte dos meios de comunicação.
Uma possível explicação para isto é o fato das taxas de infecção neste "segmento" terem começado a aumentar mais tardiamente.

Além disso, o fato deste não ser o padrão de transmissão predominante nos Estados Unidos da América e na Europa Ocidental, mesmo o sendo em regiões da África e no Caribe, parece estabelecer, equivocadamente, em nosso meio, os aspectos considerados relevantes (OMS, 1990; Winkelstein \& Johnson, 1990).

Embora os heterossexuais tenham passado a integrar os assim denominados "grupos de risco" apenas em 1984, sabe-se, hoje, que este modo de transmissão é o predominante, considerando-se a epidemia em seu contexto mundial, tendo assumido proporções crescentes em diversas regiões, como na América Latina, nos EUA e na Ásia (Haverkos \& Edelman, 1988; Piot et al., 1990; Quinn et al., 1990; Holmes et al., 1990).

A partir da Tabela 2, pode-se observar que, entre os adultos, o principal modo de transmissão, até setembro de 1992, no Brasil, foi o contato sexual, que somou, entre as duas categorias (homens homo/bissexuais e contato heterossexual), $61 \%$ dos casos.

Em nosso país, a razão homem:mulher quanto ao número de casos notificados da doença caiu de 121:1, em 1984, para 5:1, em 1991, o que pode estar indicando um aumento da transmissão heterossexual a partir de parceiros bissexuais ou de UDIs (Castilho et al., 1992a; MS, 1992). No entanto, não se pode negligenciar o fato de que, no período 1988-1990, dentre as mulheres infectadas, $41,1 \%$ eram UDIs e menos de $30,0 \%$ eram parceiras de homens bissexuais (Castilho et al., 1992a).

Fato relevante foi observado em relação à menor sobrevida dos doentes contaminados através de contato heterossexual frente àquela apresentada pelos infectados através de contato homossexual (Chequer et al., 1992a).

Sabe-se, também, que a proporção de casos decorrentes de transmissão homossexual vem apresentando uma queda sistemática de 1980$1986(46,9 \%)$ para $1992(22,4 \%)$, enquanto a proporção de casos decorrentes de transmissão heterossexual vem apresentando comportamento inverso, passando de 5,0\%, em 1980-1986, para 22,0\%, em 1992 (MS, 1992). 
TABELA 2. Número e Proporção de Casos de AIDS Segundo Categoria de Exposição e Grupo Etário Brasil, 1980-1990

\begin{tabular}{|c|c|c|c|c|c|c|}
\hline \multirow{2}{*}{ Categoria de Transmissão } & \multicolumn{2}{|c|}{ Adultos/Adolescentes } & \multicolumn{2}{|c|}{ Menores de 15 anos } & \multicolumn{2}{|c|}{ Total } \\
\hline & $\mathrm{N}$ & $\%$ & $\mathrm{~N}$ & $\%$ & $\mathrm{~N}$ & $\%$ \\
\hline \multicolumn{7}{|l|}{ Homens homossexuais/ } \\
\hline bissexuais & 12832 & 44,4 & 4 & 0,3 & 12842 & 42,7 \\
\hline UDI & 6543 & 22,7 & 23 & 2,0 & 6566 & 21,9 \\
\hline Hemofílicos/Alt. coag. & 413 & 1,4 & 184 & 16,1 & 597 & 2,0 \\
\hline Receptores de transfusão & & & & & & \\
\hline de sangue & 1165 & 4,0 & 180 & 15,7 & 1345 & 4,5 \\
\hline Contato heterossexual & 4787 & 16,6 & 3 & 0,3 & 4790 & 15,9 \\
\hline Perinatal & - & - & 634 & 55,5 & 634 & 2,1 \\
\hline Outro/desconhecido & 3149 & 10,9 & 115 & 10,1 & 3264 & 10,9 \\
\hline Total & 28895 & 100,0 & 1143 & 100,0 & 30038 & 100,0 \\
\hline
\end{tabular}

Fonte: Ministério da Saúde. Aids. Boletim Epidemiológico, ano V, no 7, 1992.

Nas mulheres com 15 anos de idade ou mais, 53,0\% dos casos de AIDS notificados em 1992 foram devidos à transmissão heterossexual e $37,1 \%$, devidos à transmissão sanguínea, sendo $31,0 \%$ por uso de drogas injetáveis. No sexo masculino, estas proporções foram de $16,4 \%$ e $27,1 \%$, respectivamente, sendo $24,6 \%$ devido ao uso de drogas injetáveis (MS, 1992).

Estudo realizado por Chequer et al. (1992b) mostrou que $4,4 \%$ dos pacientes que foram atendidos em clínicas de doenças sexualmente transmissíveis no Brasil, em 1991, eram soropositivos para o HIV-1 e que o seu nível geral de conhecimento sobre a doença era sofrível, além de apresentarem comportamentos considerados de alto risco.

Tais fatos tornam-se ainda mais relevantes se observarmos os resultados do estudo de Castilho et al. (1992b). Os autores mostram que ocorreram mudanças significativas no comportamento sexual de casais após o conhecimento da soropositividade do homem, provavelmente devido ao aconselhamento pós-teste, que reforça e/ou esclarece aspectos que as precárias campanhas nacionais não têm enfatizado devidamente, significando que é possível obter respostas eficazes em relação à prevenção. Basta, para isso, que seja dada maior ênfase à transmissão heterossexual do vírus e que sejam efetuadas intervenções efetivas.

\section{COMENTÁRIOS FINAIS}

As mudanças que vêm ocorrendo no quadro epidemiológico da AIDS e a situação ainda imprecisa desta recente pandemia revelam a impossibilidade de se restringir a doença a grupos específicos ou de se apontar um único grupo como principal responsável pela infecção/doença em nosso país.

Acreditamos que a compreensão desta situação, em contínua mudança, seja fundamental para o desenvolvimento de novas estratégias preventivas frente à epidemia.

\section{RESUMO}

BASTOS, F. I.; BOSCHI-PINTO, C.; TELLES, P. R. \& LIMA, E. M. 0 Não-dito

da A IDS. Cad. Saúde Públ., Rio de Janeiro, 9 (1): 90-96, jan/mar, 1993.

Os autores historiam a evolução da epidemia de AIDS no Brasil do ponto de vista da construção de representações sociais acerca dos segmentos envolvidos na transmissão da doença. Enfatizam a necessidade de desmistificar a correlação simplista AIDStransmissão homossexual. Procuram retirar do relativo esquecimento o papel dos usuários de drogas injetáveis e da transmissão 
heterossexual na disseminação da doença, "segmentos" cuja relevância epidemiológica, crescente em nosso meio, não se tem feito acompanhar da devida atenção, seja por parte dos meios de comunicação, seja pelos órgãos formuladores de políticas de saúde.

Palavras-Chave: AIDS; Transmissão Heterossexual do HIV; Transmissão do HIV entre Usuários de Drogas Injetáveis (UDIs)

\section{REFERÊNCIAS BIBLIOGRÁFICAS}

BARATTA, A., 1992. Fundamentos ideológicos da atual política criminal sobre drogas. In: Só Socialmente (F. I. Bastos \& O. Gonçalves, orgs.), pp. 35-50, Rio de Janeiro: Relumé Dumará.

BERNARD, R. P., 1992. AIDS-FEEDBACK through 09/1991 [AF 09 91/c], p. 12, Genebra: Organização Mundial da Saúde.

CARRARA, S. \& MORAES, C., 1987a. Um vírus só não faz doença. Cadernos do Instituto de M edicina Social, 1: 96-123. 1987b. Um mal de folhetim. Cadernos do Instituto de Medicina Social, 2: 79-94.

CASTILHO, E. A.; SZWARCWALD, C. L. \& BOSCHI-PINTO, C., 1992a. AIDS: a Brazilian case-study. In: Adult M ortality in Latin America (A. Lopez, org.). International Union for the Scientific Study of Population (IUSSP). Liege, Bélgica.

CASTILHO, E. A.; BOSCHI, C.; GUIMARÃES, M. D. C.; SERENO, A.; CAVALCANTE, S.; HART, H.; LIMA, L. A. \& MORGADO, M., 1992b. Sexual behavior change after knowledge of HIV-1 test status. In: VIII International Conference on AIDS, Abstracts, poc 4359, p. C304, Amsterdã.

CDC (Center for Disease Control), 1992. HIV/AIDS Surveillance Report - Atlanta, GA, April.

CHEQUER, P.; HEARST, N.; HUDES, E. S. CASTILHO, E.; RUTHERFORD, G.; LOURES, L. \& RODRIGUES, L., 1992a. Determinants of survival in adult Brazilian AIDS patients, 19821989. AIDS, 6: 483-487.

CHEQUER, P.; HEARST, N.; CASTILHO E. A.; RODRIGUES, L.; PERALTA, M.; LANDMANN, C.; BENZAKEN, A.; SANTOS, E.; ONAGA, E.; RAMOS, M. \& BUENO, L., 1992b. HIV antibody prevalence in STD patients of five state capitals in Brazil. In: VIII International Conference on AIDS, A bstracts, poc 4296, p. C234, Amsterdã.
COSTA, J. F., 1991a. Os amores que não se deixam dizer. Cadernos de Psicanálise, 7: 57-71.

, 1991b. Por uma psicanálise humanamente útil. In: A nuário Brasileiro de Psicanálise, pp. 13-23, Rio de Janeiro: Relumé Dumará.

DANIEL, H. \& PARKER, R., 1990. AIDS - A terceira epidemia. São Paulo: Iglu.

DIAS, M. B. S.; LIMA, J. N.; ABREU, W. B.; AOKI, F. M.; GONÇALES, N. \& PEDRO, R. J., 1989. Follow-up of groups at risk for HIV infection. In: V International Conference on AIDS, A bstracts, T.G.P. 14, p. 317, Montreal.

EUROPEAN CENTRE FOR THE EPIDEMIOLOGICAL MONITORING OF AIDS, 1991. AIDS surveillance in Europe. Quarterly Report, 13, Geneva: WHO, September.

FRY, P., 1982. Para Inglês Ver. Rio de Janeiro: Zahar.

GIANNA, M. C.; CHEQUER, P.; CASTILHO, E.; KALICHMAN, A.; PINTO, V. \& RODRIGUES, L., 1989. Profile of IVDU in an AIDS reference center, São Paulo, Brazil, 1988. In: VI International Conference on AIDS, Abstracts, Th. C. 713, p. 303, São Francisco.

HAVERKOS, H. W. \& EDELMAN, R., 1988. The epidemiology of Acquired Immunodeficiency Syndrome among heterosexuals. Journal of the American Medical Association, 260: 19221929.

HOLMES, K. K.; KARON, J. M. \& KREISS, J. K., 1990. The increasing frequency of heterosexually acquired AIDS in the United States, 1983-1988. American Journal of Public Health, 80: 858862.

LEITE, E. A.; HAMERSCHLAK, N.; SZTERLING, L.; CARVALHO, M. B.; LIMA, M. P. J. S. \& BELLUCCI, S. B. B., 1989. HIV infection in IV drug abusers. In: V International Conference on AIDS, Abstracts, D 709, p. 809, Montreal.

LIMA, M. P. S.; ROCHA, M. D. C.; TANIBATA, P. R.; MIELLE, A.; FERREIRA, R. P. \& LORENZETTI, T. M., 1989. Profile of users of the drug IV in the periphery of Campinas. In: V International Conference on AIDS, A bstracts, D. 604, p. 792, Montreal.

Mac RAE, E., 1990. A Construção da Igualdade. Campinas: Unicamp.

MESQUITA, F.; MOSS, A. R.; REINGOLD, A. L.; BUENO, R. C.; PAES, G. T.; CARVALHO, H. B. \& RUIZ, M. A., 1992. HIV antibody seroprevalence among IVDUs in the city of Santos São Paulo State - Brazil. In: VIII International Conference on AIDS, Abstracts, puc 8140, p. 176, Amsterdã. 
MS (Ministério da Saúde), 1992. Divisão Nacional de Doenças Sexualmente Transmissíveis (DST / AIDS). Brasília. AIDS - Boletim Epidemiológico, ano $\mathrm{V}, \mathrm{n}^{\circ} 7$, p. 7 .

OMS (Organização Mundial da Saúde), 1990. Boletim de AIDS, janeiro, v. 7, p. 5.

PARKER, R. G., 1991. Corpos, Prazeres e Paixões. Rio de Janeiro: Bestseller.

PIOT, P.; LAGA, M.; RYDER, R., 1990. The global epidemiology of HIV infection: continuity, heterogeneity, and change. Journal of Acquired Immune Deficiency Syndromes, 3: 403-412.

QUINN, T. C.; NARAIN, J. P. \& ZACARIAS, F. R. K., 1990. AIDS in the Americas: a public health priority for the region. AIDS, 4: 709-724.

SANTOS, L. A. C.; MORAES, C. E. \& COELHO, V. S. P., 1991. A hemoterapia no Brasil de 64 a 80. Revista Physis, 1: 161-182.

, 1992. Os anos 80 - a politização do sangue. Revista Physis, 1: 107-150.
SCHILLER, N. G., 1992. What's wrong with this picture? The hegemonic construction of culture in AIDS research in the United States. Medical Anthropology Quarterly, 3: 237-254.

TELLES, P. R.; BASTOS, F. I.; LIMA, E. S.; FRIEDMAN, S. R. \& DESJARLAIS, D. C., 1992. HIV-1 epidemiology among IDUs in Rio de Janeiro, Brazil. In: VIII International Conference on AIDS, Abstracts, poc 4265, p. C289, Amsterdã.

VALLINOTO, T. C., 1991. A construção da solidariedade. Tese de Mestrado, Rio de Janeiro: Escola Nacional de Saúde Pública.

WINKELSTEIN Jr., W. \& JOHNSON, A. M., 1991. Epidemiology: overview. In: AIDS 1990. A year in review (J. A. Levy, ed.), pp. S59-S97, Philadelphia: Current Science. 